\title{
Expert Maintenance System for Life Cycle Assessment of Heavy Machinery
}

\author{
Prakash Kumar ${ }^{1}$, Piush Raj ${ }^{2}$ \\ ${ }^{1}$ Assistant Professor, Production Engineering Department, B. I. T. Sindri, Dhanbad-828123 \\ ${ }^{2} J u n i o r$ Manager, Project, HEC, Ranchi.
}

\begin{abstract}
The paper provides a brief overview of the research areas of heavy machinery through maintenance models. It is not meant to be a complete review of maintenance models but rather as an informative introduction to important maintenance models.

The paper is concerned with the basic preventive maintenance policies arising in the context of heavy machinery maintenance. The investigation in this paper relates mainly to develop preventive replacement model and fault tree analysis for the horizontal boring machine and to reduce the down-time of the machine.

Simple but practically important preventive maintenance optimization models, which involve age replacement and block replacement, are reviewed in the framework of the expert maintenance policy.

The study was carried out in a mechanical workshop of an engineering construction company in a private sector. There are different machines and hand tools such as Bending machine, CNC Profile cutting machine, vertical turning and boring, horizontal turning and Boring, Gear hobbling machine, Lathe machine, Milling Machine, Plano miller, Planner machine, Grinding Machine, Press, Shearing Machine, hammers and area repair shops equipments. Since the concern was on machining of construction equipment so the study was concentrated on Horizontal \& Vertical Turing and Boring.

The study was carried out with the following objectives -

To develop the methodology for maintenance policies in a job order shop industry due to ill maintenance and to apply fault tree analysis for the development of maintenance policies and expert system.

The paper also analyzes the facts of a case study in order to find optimal cost of repair/replacement for finite horizon replacement model when preventive maintenance is imperfect. The maintenance technique used for the analysis of this case study is simple and is based on the statistical calculation of total down time, mean time to repair, mean time between failure and fault tree analysis. Based on these studies an expert system is developed. Financial impact of these studies are also taken into consideration.
\end{abstract}

Keywords: Expert system, Fault Tree Analysis, Markov Model, MTBF, MTTR, Preventive Maintenance

\section{Introduction}

Maintenance function is one of the most important functions of any industry. To keep any industry on wheel and in path of progress, this function can not be neglected. Maintenance policies are followed to reduce the incidence of system failure or to return a failed system to the operating state. No matter how carefully a system may be designed and manufactured it is impossible to be absolutely certain that it will not fail. Thus in order to calculate the availability of any system to survive for a specified period of time followings have to considered : Readiness or availability of the system, and Mission availability.

Maintenance cost is another key factor and that must be taken into consideration. Maintenance cost can be minimized by avoiding unnecessary maintenance but in the meanwhile, not missing the required maintenance. Increasing equipment reliability by reducing the equipment failure and production down time is the concept behind lean maintenance and to achieve it, total planned maintenance is necessary.

\section{The Maintenance Strategy}

Maintenance can be considered as a combination of actions carried out to keep a facility in such a condition that it may be utilized at its optimum.

We categories the whole machine (HBM) faults in three parts: Mechanical, Electrical and Lubrication. These three faults are again categorized in different sub-parts that can be shown in the figure of Fault Tree Analysis.

However, generally a preventive maintenance policy is adopted to minimize the possibility of an anticipated production interruption or breakdown. While developing the maintenance policy we must consider FTA, MTTR, MTTF and Total Production Loss due to Failure for Horizontal Boring Machine. 
III. Calculation of MTTR,MTTF and Total Production Loss per Failure

For this we take only one example of MTTR, MTTF and Total Production Loss per Failure calculation and also plot the graph for this fault that is Miscellaneous Mechanical Fault. Similarly, we calculate Miscellaneous Electrical Fault, Vertical Slide Problem, Connector Problem, Telescopic Brush Problem and Lubrication Problem from the data.

Fig 1. Fault Tree Analysis of Horizontal Boring Machine (158)

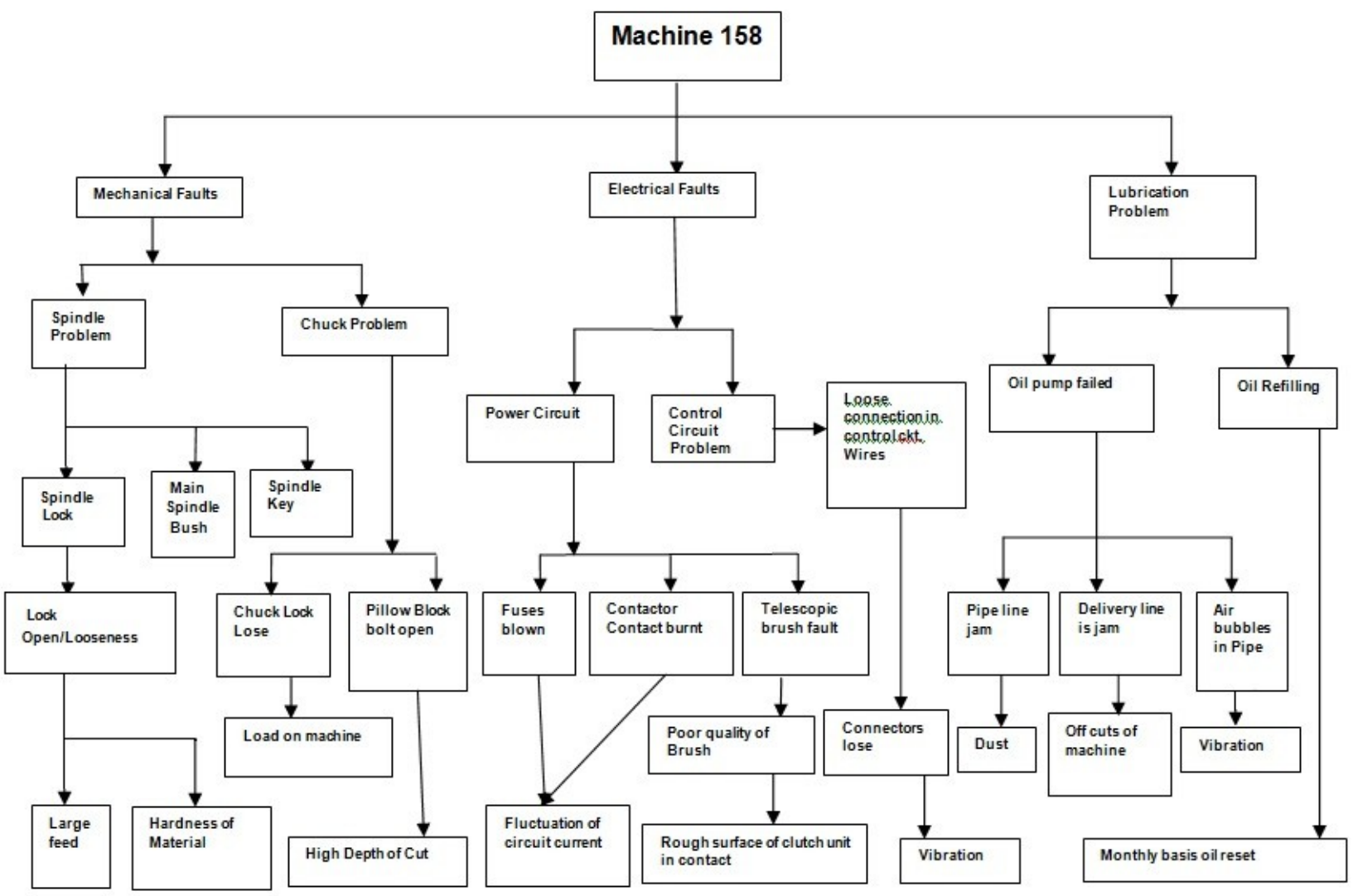

Graph1. Total down time versus Period of fault due to Miscellaneous Mechanical Problem

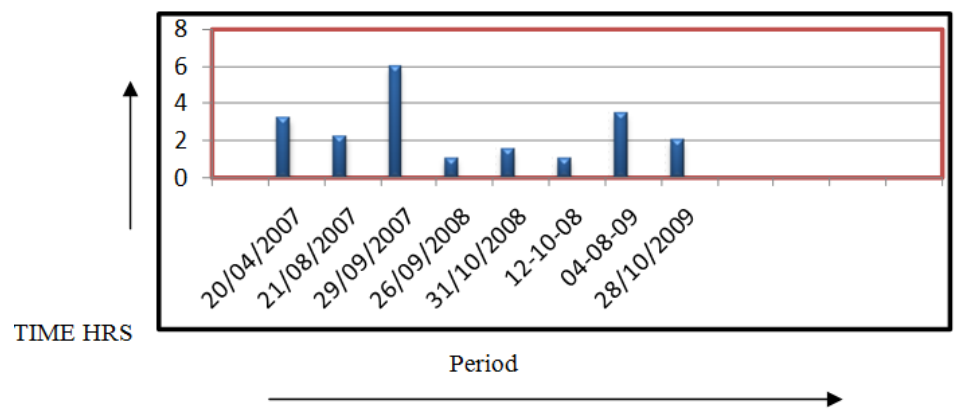

Mean Time to Repair

$$
\begin{gathered}
\text { M.T.T.R }=\frac{1(3.25+2.25+6+1+1.5+1+3.5+2)}{8} \text { hours } \\
\text { M.T.T.R }=\mathbf{2 . 5 6} \text { hours }
\end{gathered}
$$


Graph2. Cost versus Period of fault due to Miscellaneous Mechanical Faults.

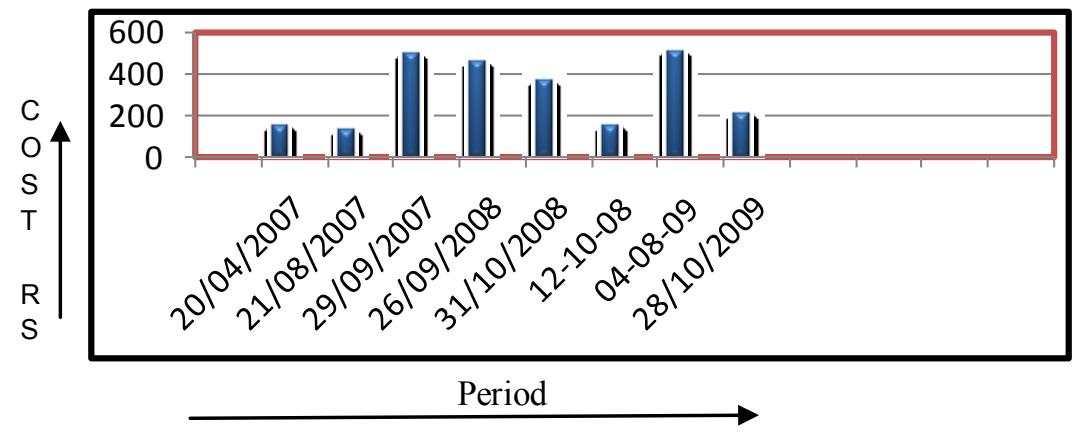

Average cost per failure $=$ Rs 301.98

Total Production Loss $=$ Rs $301.98+(2.5 \mathrm{Hr} \times 1200 \mathrm{Rs} / \mathrm{Hr})$

Total Production Loss $=$ Rs 3301

TABLE I

Calculation of Mean Time to Failure due to Miscellaneous Mechanical Problem

\begin{tabular}{|c|c|c|c|c|}
\hline SI No. & $\mathbf{1}^{\text {st }}$ Failure & $\mathbf{2}^{\text {nd }}$ Failure & Total Time (Days) & $\begin{array}{c}\text { Total Time } \\
\text { (Weeks) }\end{array}$ \\
\hline 1 & & & & 17 \\
\hline 2 & $20-04-07$ & $21-08-07$ & 120 & 4 \\
\hline 3 & $21-08-07$ & $29-09-07$ & 30 & $52^{*}$ \\
\hline 4 & $29-09-07$ & $26-09-08$ & 365 & 4 \\
\hline 5 & $26-09-08$ & $3-10-08$ & 30 & 6 \\
\hline 6 & $31-10-08$ & $10-12-08$ & 45 & 17 \\
\hline 7 & $10-12-08$ & $8-04-09$ & 120 & 20 \\
\hline
\end{tabular}

Total Week- 120

The data marked is outlier data.

$$
\text { M.T.T.F }=1 / 7(\text { Total week })
$$

\author{
M.T.T.F $=17.15$ Weeks \\ New M.T.T.F $=1 / 6(17+4+4+6+17+20)$ \\ New M.T.T.F $=11.33$ Weeks
}

\title{
IV. Development Of An Expert System Based On Calculations Of M.T.T.F
}

An expert system (ES), also called a knowledge based system, can be defined as an intelligent computer programme that has the capability to solve difficult real life problems using a knowledge base and inference procedures. Expert system utilizes a knowledge base containing facts, data, definitions and assumptions. They also have the capacity for a heuristic approach, i.e, making good judgment by discovering and revelation and making good guesses, just as an expert would. The knowledge required to perform this task is called the domain of the system. The knowledge base is expressed in computer course usually in the form of if- then rules, with a series of questions. The mechanism to use these rules to solve the problem is called an inference engine. Expert system can also communicate with other computer software packages.

To analyze the data of mean time to failure of the different components, we develop a model or a system which can be utilized in preventive maintenance of the machine. Even though for the machine breakdowns, we calculate the mean time to repair which becomes vital while repairing the machine. So, we put together the various M.T.T.F data in table II and categorize them according to the time before which preventive maintenance should be done.

V. Table II Comparison of M.T.T.F. Due to Out Lier Data

\begin{tabular}{|c|c|c|c|}
\hline SI. No. & Description & M.T.T.F (Weeks) & M.T.T.F(New) (Weeks) \\
\hline 1 & Miscellaneous Electrical Fault & 9.5 & 7 \\
\hline 2 & Vertical Slide Problem & 14 & 3.66 \\
\hline 3 & Spindle Problem & 14.22 & 14.22 \\
\hline 4 & Connector Problem & 14.75 & 11.71 \\
\hline 5 & Telescopic Brush Problem & 16.5 & 16.5 \\
\hline 6 & Miscellaneous Mechanical Fault & 17.14 & 11.33 \\
\hline 7 & Lubrication Problem & 22.2 & 19.5 \\
\hline
\end{tabular}


Now as there are seven parts we can categorize them when preventive maintenance has to be done as per schedule,

A less than 10 Weeks. Category A kind of preventive maintenance has to be done during first 10 weeks.

$11<\mathrm{B}<15$ Weeks. Category B, has to be taken care of between 10 to 15 weeks of different parts of the machine.

$\mathrm{C}$ more than 16 Weeks. Category $\mathrm{C}$ has to be taken care of preventive maintenance schedule which has to be maintained for more than 16 weeks.

Now, elaborating each of the categories and developing the Expert System considering the fault tree.

\section{A. Condition A: Less Than 10 Weeks}

If the number of weeks is less than 10 and if Clutch is not working properly, If Spindle movement is restricted and if Vertical slide keys are misplaced.

Then check the vertical slide of the machine, electrical connections and control cables of the machine, check the current fluctuations, and the amount of vibration occurring while machining of the job piece. Else machine will operate positively.

\section{B. Condition B: Between $11^{\text {th }}$ And $15^{\text {th }}$ Week of Preventive Maintenance}

If the number of weeks of preventive maintenance schedule is between the $11^{\text {th }} \& 15^{\text {th }}$ week and If connector contacts are burnt, If fuses are blown, If chuck lock loosens,

If pillow block bolts are opened/loosed, if lead screw lock nut is removed, If spindle lock loosens, If spindle main bush wears out and If spindle key is removed.

Then check the spindle of the machine, vertical slide of the machine. Check the Power circuit connections of the machine, and the material harness which is to be cut, also the amount of feed given to the job. Else machine will operate positively.

\section{Condition C: More Than 16 Week}

If the number of weeks of preventive maintenance schedule is more than 16 week, and

If Telescopic brush faults are coming, If contact of clutch surface is rough,

If brushes are of poor quality, If pipe line for circulation of oil is jam,

If delivery line is jam, If air bubbles are trapped in the pipe line,

If lubricants viscous properties have changed.

Then change the telescopic brush, check the lubrication system of the machine, reset the lubrication oil of the machine. Else the machine will run positively.

\section{Markov Models and Process}

The calculation of the reliability of a system with elements exhibiting dependent failure and involving repair or standby operations is a general complicated approaches that has been suggested to carry out the computations. A technique that has much appeal and works well when failure hazards and repair hazards are constantly required.

In this case the system is denoted by $\mathrm{X}$ and the components constituting the system are as $\mathrm{X}_{1}-$ Mechanical Faults, $X_{2}-$ Electrical Faults, $X_{3}$ - Lubrication Faults. This system state changes with time t. The system state $\mathrm{X}$ and time $\mathrm{t}$ are two random variables. Since the system is probability based, then the model is formulated according to Markov probability model.

In this model, we associate the state of the system with probability $\mathrm{P}_{\mathrm{ij}}$, indicating the probability of the system moving from state $\mathrm{i}$ to state $\mathrm{j}$. This probability $\mathrm{P}_{\mathrm{ij}}$ is called the transition probability. In this process, we make the fundamental assumption that the transition probability from $i$ to $j$ depends entirely on state $i$ and $j$, and is independent of all previous states except the last one that is state $i$.

\section{A. Markov Graph and Application to Subject}

It is usual to display a Markov process pictorially, and this pictorial representation is called Markov graph. A Markov graph consists of nodes and branches. The nodes represent the states that the system can be in, and the branches carry with them the respective transitional probabilities. Consider the single-element system having one of the two state stages, $s_{0}$ or $s_{1}$. Hence, the Markov graph for this will consists of nodes representing states $s_{0}$ and $s_{1}$ If the system is in state $s_{0}$ at time $t$, then it can either move to state $s_{1}$ or stay in state $s_{0}$ in the interval $\delta$ t. Therefore, there will be two branches emanating from node $s_{0}$, one going to node $s_{1}$ and the other looping back to node $\mathrm{s}_{0}$. These are shown below in fig 2 . 


\section{Fig 2 Markov Graph for two element system}

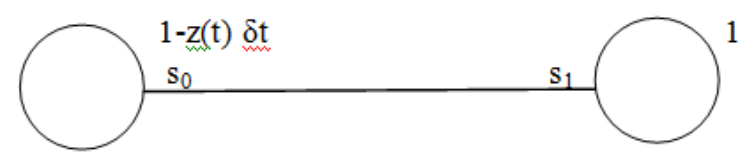

Figure above indicates Markov Graph for two element system

If the system is in state $s_{1}$ at time $t$, then, $s_{1}$ being an absorbing state, the branch from node $s_{1}$ can only be a loop coming back to node $\mathrm{s}_{1}$. The transitional probabilities are indicated along the respective branches. It should be observed that the sum of transition probabilities indicated on the branches emanating from each node is equal to unity. If we treat each node as a source of signal and the branches as lines carrying the signal with transmission coefficients equal to the transition probabilities, then we can write the difference equation for each state just by inspection. Thus, for node $\mathrm{s}_{0}$, the probabilities, then we can write the difference equation for each state just by inspection. Thus, for node $s_{0}$, the probability of being in state $s_{0}$ at time $(t+\delta t)$ is equal to the sum of the signals arriving at node $\mathrm{s}_{0}$. The sum of the signals arriving at $\mathrm{s}_{0}$ is equal to

$\mathrm{Ps}_{0}(\mathrm{t})[1-\mathrm{Z}(\mathrm{t}) \delta \mathrm{t}]=\mathrm{Ps}_{0}(\mathrm{t}+\delta \mathrm{t})$.

Similarly, for node $\mathrm{s}_{1}$, we have

$\mathrm{Ps}_{0}(\mathrm{t})[\mathrm{Z}(\mathrm{t}) \delta \mathrm{t}]+\mathrm{Ps}_{1}(\mathrm{t}) \cdot 1=\mathrm{Ps}_{1}(\mathrm{t}+\delta \mathrm{t})$.

Let us now construct the Markov graph for a three element system $\mathrm{X}$ considering $\mathrm{X}_{1}$ - Mechanical Faults, $X_{2}$ - Electrical Faults, $X_{3}$ - Lubrication Faults as components of the system X (M/c 158).The system consisting of three elements $X_{1}, X_{2}$, and $X_{3}$ and has seven possible states,

$\mathrm{S}_{0}=\mathrm{X}_{1} \mathrm{X}_{2} \mathrm{X}_{3}$

$\mathrm{S}_{1}=\mathrm{X}_{1} \overline{\mathrm{X}}_{2} \mathrm{X}_{3}$

$\mathrm{S}_{2}=\mathrm{X}_{1} \mathrm{X}_{2} \overline{\mathrm{X}_{3}}$

$\mathrm{S}_{3}=\mathrm{X}_{1} \mathrm{X}_{2} \mathrm{X}_{3}$

$\mathrm{S}_{4}=\mathrm{X}_{1} \overline{\mathrm{X}_{2}} \frac{\overline{\mathrm{X}_{3}}}{\mathrm{X}_{3}}$

$\mathrm{S}_{5}=\mathrm{X}_{1} \mathrm{X}_{2} \mathrm{X}_{3}$

$\mathrm{S}_{6}=\mathrm{X}_{1} \overline{\mathrm{X}}_{2} \mathrm{X}_{3}$

$\mathrm{S}_{7}=\mathrm{X}_{1} \overline{\mathrm{X}}_{2} \overline{\mathrm{X}_{3}}$

Hence the graph will consists of eight nodes $\mathrm{S}_{0}, \mathrm{~S}_{1}, \mathrm{~S}_{2}, \mathrm{~S}_{3}, \mathrm{~S}_{4}, \mathrm{~S}_{5}, \mathrm{~S}_{6}, \mathrm{~S}_{7}$. From node $\mathrm{S}_{0}$, transitions can take place to states $\mathrm{S}_{1}, \mathrm{~S}_{2}, \mathrm{~S}_{3}, \mathrm{~S}_{4}, \mathrm{~S}_{5}, \mathrm{~S}_{6}, \mathrm{~S}_{7}$

Fig 3 shows Markov loops and nodes for three element system

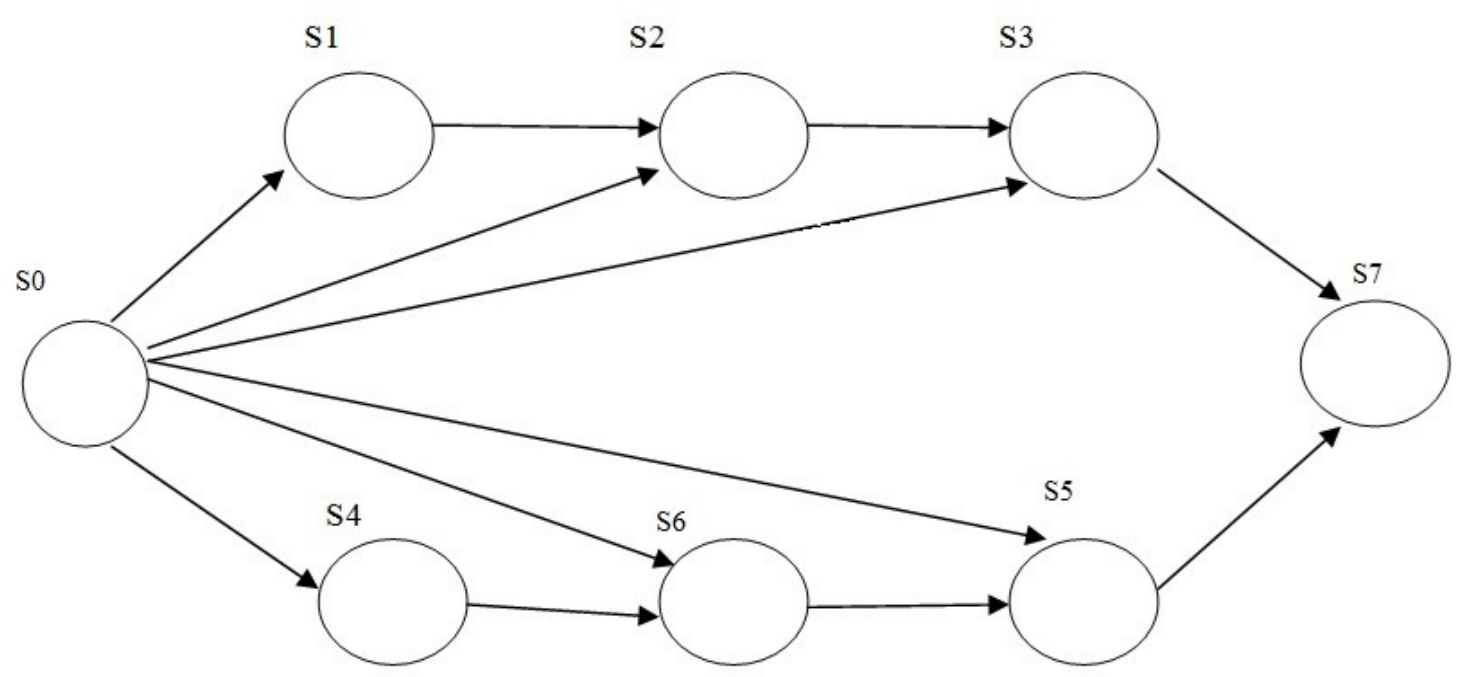

Hence there are six branches and seven loops (Miscellaneous Electrical Fault, Vertical Slide Problem, Spindle Problem, Connector Problem, Telescopic Brush Problem, Miscellaneous Mechanical Fault \& Lubrication Problem) emerging from node $\mathrm{S}_{0}$. These are indicated in fig 3. In the Markov process we have made fundamental assumption about the transition probability $\mathrm{P}_{\mathrm{ij}}$, namely, this probability of transition from $\mathrm{i}$ to $\mathrm{j}$ for any moment $t_{0}$ depends only on states $i$ and $j$ and does not depend on when and how the system reaches state $i$. In other words, for a stabilized condition the transition does not depend on path. Thus, we obtain from the Markov Model that machine 158, will have states of failure of mechanical, electrical and lubrication existing 
either one at a time or two at a time as expressed by figure shown above. The transition state take place i.e. one fault get repaired once and come back to original state $\mathrm{s}_{0}$ or, two faults (taking place at a time) get repaired and come back to its initial state.

\section{Conclusion}

The paper shows how to develop an expert system for heavy machinery through various maintenance models. The maintenance models are recommended in situations where it is difficult to develop an analytical solution.

The failure data has been analyzed for three years. The historical data (card/log book) were taken for machine 158. Inspection was done on the shop floor before commencement of work. This includes checking of belt, fuses, contactors contact, check nuts, key ways etc.

Routine Repair - Routine repair includes periodically checking up of machine parts which may lead to breakdown of machine. These are categorized as checking of oil sealing, proper greasing of lubricating parts, checking of valves. This is indicated by ' $\mathrm{O}$ '.

Medium Repair - Medium repair focuses on those repair, which are present in machine but still the machines runs. If these are not attended in short time then it may lead to major repair. These are indicated generally by ' $M$ '.

Capital Repair - When the machine require investment after a few medium repairs than it is termed as capital repair. It requires investment of capital to the machine. This is denoted by ' $\mathrm{K}$ '.

Also plant preventive maintenance schedule for this horizontal boring machine is 120 and having weight in between 10T \& 100T. Hence on the basis of this following preventive schedule is followed:

$\mathrm{K}-\mathrm{O}_{1}-\mathrm{O}_{2}-\mathrm{O}_{3}-\mathrm{M}_{1}-\mathrm{O}_{4}-\mathrm{O}_{5}-\mathrm{O}_{6}-\mathrm{M}_{2}-\mathrm{O}_{7}-\mathrm{O}_{8}-\mathrm{O}_{9}-\mathrm{C}_{1}-\mathrm{O}_{10}-\mathrm{O}_{11}-\mathrm{O}_{12}-\mathrm{M}_{3}-\mathrm{O}_{13}-\mathrm{O}_{14}-\mathrm{O}_{15}-\mathrm{C}_{2}-\mathrm{O}_{16}-\mathrm{O}_{17}-\mathrm{O}_{18}-\mathrm{M}_{4}-$ $\mathrm{O}_{19}-\mathrm{O}_{20}-\mathrm{O}_{21}-\mathrm{M}_{5}-\mathrm{O}_{22}-\mathrm{O}_{23}-\mathrm{O}_{24}-\mathrm{M}_{6}-\mathrm{O}_{25}-\mathrm{O}_{26}-\mathrm{O}_{27}-\mathrm{K}$

\section{Future Scope}

1. By using different maintenance models, we can efficiently build a simulation Meta model.

2. The use of meta model allows us to generalize simulation output.

3. Sensitivity analysis can equally be conducted within the bounds of the model without re-running costly simulation programs.

4. Regression Meta models can also be developed.

\section{References}

[1] Adhammar Et.al, Preventive Management/Essential care Condition monitoring

[2] D.W.Rolston, Principles of Artificial Intelligence and Expert Systems Development, McGraw-Hill, 1988

[3] Jardine AKS, Maintenance, Replacement \& Reliability, Pitman Publication

[4] Jim Parentzas, A Wab- Based controlled By a Hybrid expert System.

[5] L.S.Srinath, Reliability Engineering Affiliated East-West Press Private Limited New Delhi.

[6] Md. Ben-Daya, Salih O. Dufffuaa, Abdul Raouf Maintenance, Modeling\& Optimization

[7] Prakash Kumar, Rajak A.K.,2014,Advanced Functional Maintenance Management for Mining Excavator, in an International journal of Mechanical Engineering \& Technology (IJMET) Volume 5, Issue 4, April (2014), pp. 199-205.

[8] Prakash Kumar, Srivastava R.K., 2014, Development of Condition Based Maintenance Architecture for Optimal Maintainability of Mine Excavators" in an International Organization of Scientific Research- Journal of Mechanical and Civil Engineering (IOSRJMCE). Volume 11, Issue 3 Ver. V PP 18-22.

[9] Probabilistic Risk Assessment and Management for Engineers \& Scientists, IEEE Press (2 ${ }^{\text {nd }}$ Edition), 1996, EJ Henley \& H. Kumamoto 1996.

[10] Reliability and Fault Tree analysis, Conference on Reliability and Fault tree analysis; UC Berkeley; SIAM Pub;

[11] Reliability and Risk Assessment, Longman Scientific \& Technical 1993, J.D.Andrew1993.

[12] R.Keith Mobley, Lindley R. Higgins \& Darrin J. Wikoff, 2008, Maintenance Engg. Handbook, $2^{\text {nd }}$ edition. 